\title{
Unilateral vocal cord palsy in a patient with jugular foramen schwannoma
}

\author{
Hong Kong Med J 2021;27:303.e1-2 \\ https://doi.org/10.12809/hkmj208791
}

WK Wong, CY Cheng, WC Cheng *

\begin{abstract}
A 74-year-old man presented with a history of Laryngoscopy revealed left vocal cord palsy (Fig 1 ). Non-contrast computed tomography (CT) showed no pulmonary or neck lesion, but one lobulated, centrally low attenuating mass. T2-weighted magnetic resonance images confirmed a $3.8-\mathrm{cm}$ soft tissue mass with heterogeneous enhancement in the left cerebellopontine angle. In both images there was
\end{abstract}

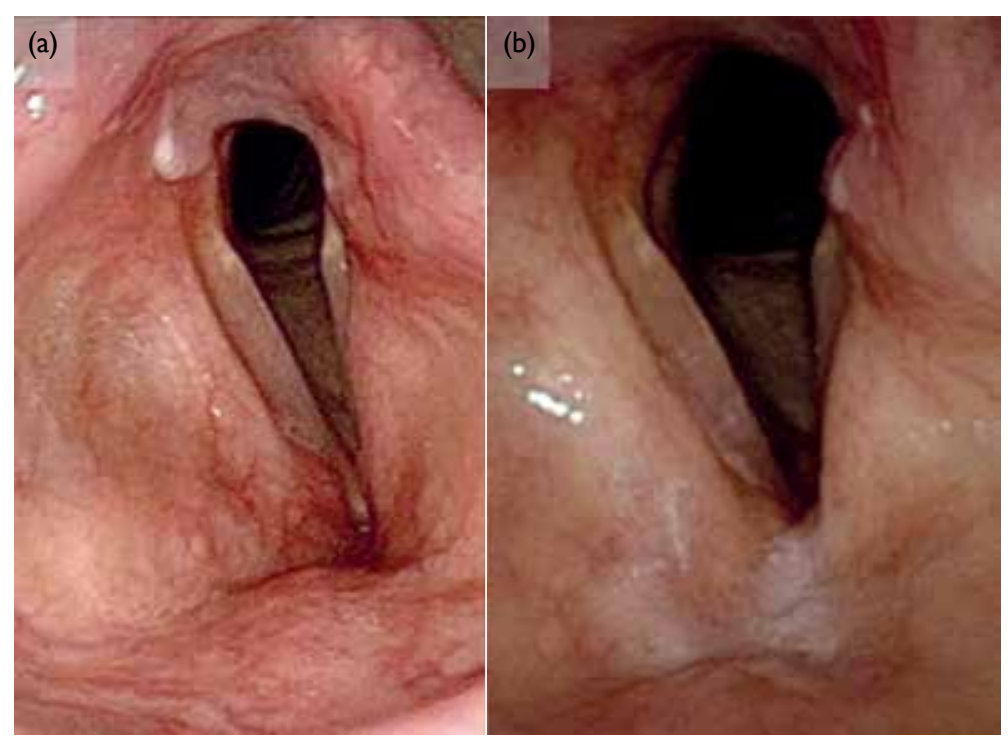

FIG I. Laryngoscopic examination showing (a) left vocal cord palsy before surgery and (b) improvement after surgery

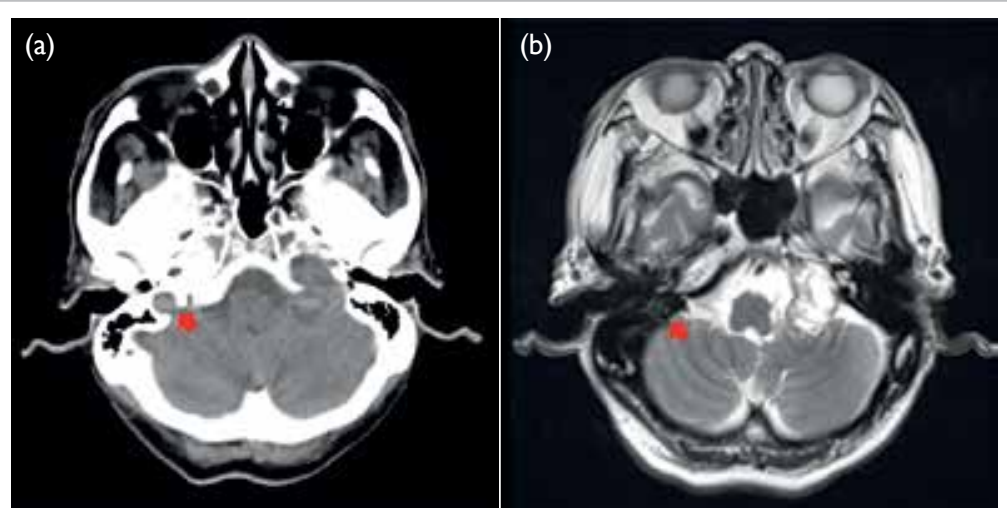

FIG 2. (a) Non-contrast computed tomography and (b) T2-weighted magnetic resonance image showing a soft tissue mass in the left cerebellopontine angle. The arrows indicate the location of the normal right jugular foramen in both figures extension of the mass through an expansion of the left jugular foramen, widened left internal acoustic meatus and skull base destruction (Fig 2), compatible with jugular foramen tumour. Subsequently the patient developed progressive globus sensation, tinnitus, left sternocleidomastoid muscle wasting, and left neurosensory hearing loss. The tumour was resected via a retrosigmoid approach. Surgical assessment of the tumour revealed its origin from cranial nerves IX and X and compression of cranial nerves VIII and XI. The histopathological diagnosis with immunohistochemistry staining was that of schwannoma. Postoperative imaging demonstrated adequate resection of the tumour. Improvement in swallowing and hearing function test from severe to moderate impairment were noted postoperatively.

\section{Discussion}

Schwannoma is a benign tumour of the nerve sheath. The most common intracranial schwannoma is vestibular schwannoma (acoustic neuroma) that arises from cranial nerve VIII. ${ }^{1}$ Jugular foramen schwannomas, which mainly arise from cranial nerves IX and X, account for around 3\% to $4 \%$ of all intracranial schwannomas. They are more prevalent in women and occur between the third and sixth decades of life. Clinical presentation is variable because of their slowgrowing nature and proximity to other cranial nerves. Symptoms appear when the tumour is sufficiently large and most commonly consist of hearing loss, tinnitus, dysphagia, ataxia, and hoarseness. Other symptoms include dysarthria, dysphonia, aspiration, vertigo, dizziness, shoulder weakness, and headache. ${ }^{2}$ Differential diagnoses of unilateral vocal cord palsy are usually divided into malignancies of the lung and the neck and non-malignant lesions of traumatic, neurological, inflammatory or infectious origin. A CT scan is indicated to evaluate the more common pulmonary malignancies and to ensure presence of a rarer lesion along the course of the recurrent laryngeal and vagus nerve up to the skull base is not missed, especially if signs and symptoms of multiple cranial nerve involvement are present. ${ }^{3}$

Imaging studies can help differentiate vestibular and lower cranial nerve schwannomas, meningioma, glomus jugulare paraganglioma, ependymomas, and metastatic tumour. Imaging findings of jugular foramen schwannoma include scalloped and sclerotic expansion of the temporal bone instead of a lytic 
pattern. On magnetic resonance imaging, the lesion is T1 iso- or hypo-intense or T2 iso- or hyper-intense with gadolinium enhancement, whereas on $\mathrm{CT}$, it is isodense to brain parenchyma and enhanced with contrast. ${ }^{4}$ The site of origin is classified as cisternal, foraminal (intraosseous) or extracranial. However, it is difficult to ascertain the exact site radiologically and clinically because of the variable location and nerve involvement. Assessment of the nerve root during surgery is required to confirm the origin of the tumour. The selection of surgical approach for treatment is determined by the location, pattern and extension of the tumour, the degree of bone destruction/erosion and the neurological and preoperative hearing status. ${ }^{5}$ Risks of operation include damage to other cranial nerves, especially facial nerve palsy, and incomplete removal of the tumour leading to recurrence.

\section{Author contributions}

Concept or design: All authors.

Acquisition of data: WK Wong, CY Cheng.

Analysis or interpretation of data: All authors.

Drafting of the manuscript: WK Wong.

Critical revision of the manuscript for important intellectua content: All authors.

All authors had full access to the data, contributed to the study, approved the final version for publication, and take responsibility for its accuracy and integrity.

\section{Conflicts of interest}

All authors have disclosed no conflicts of interest.

\section{Funding/support}

This study received no specific grant from any funding agency in the public, commercial, or not-for-profit sectors.

\section{Ethics approval}

The patient was treated in accordance with the Declaration of Helsinki and provided consent for all investigations and procedures

WK Wong, BDS, MD

CY Cheng, MD

WC Cheng *, MD

Department of Neurosurgery, Chang Gung Memorial Hospital, Chiayi,

Taiwan

*Corresponding author: wancheng7511@yahoo.com.tw

\section{References}

1. Suri A, Bansal S, Singh M, Mahapatra AK, Sharma BS. Jugular foramen schwannomas: a single institution patient series. J Clin Neurosci 2014;21:73-7.

2. Bakar B. The jugular foramen schwannomas: review of the large surgical series. J Korean Neurosurg Soc 2008;44:28594

3. Stimpson P, Patel R, Vaz F, et al. Imaging strategies for investigating unilateral vocal cord palsy: how we do it. Clin Otolaryngol 2011;36:266-71.

4. Lee M, Tong K. Jugular foramen schwannoma mimicking paraganglioma: case report and review of imaging findings. Radiol Case Rep 2016;11:25-8.

5. Samii M, Alimohamadi M, Gerganov V. Surgical treatment of jugular foramen schwannoma: surgical treatment based on a new classification. Neurosurgery 2015;77:424-32. 